\title{
LA BARRERA ELECTORAL
}

ENRIQUE ÁLVAREZ CONDE

SUSANA GARCÍA COUSO 


\section{SUMARIO}

I. Introducción. II. La barrera electoral: 1. Principios informadores: Igualdad, Proporcionalidad y Razonabilidad. 2. Relación con los demás ELEMENTOS QUE CONFIGURAN EL SISTEMA ELECTORAL. III. LA BARRERA EN EL SIStema electoral español: 1. Elecciones Generales. 2. Elecciones Autonómicas. 3. Elecciones Locales. 4. Elecciones Europeas. IV. Referencias BIBLIOGRÁFICAS. 


\title{
LA BARRERA ELECTORAL
}

POR

\author{
ENRIQUE ÁLVAREZ CONDE \\ Catedrático de Derecho Constitucional \\ Universidad Rey Juan Carlos \\ SUSANA GARCÍA COUSO \\ Profesora Asociada de Derecho Constitucional \\ Universidad Rey Juan Carlos
}

\section{INTRODUCCIÓN}

Como dijo RAE (1977), "Los actuales conocimientos de Derecho electoral no son ni de alcance muy general ni de contenido enteramente fiel. No hay tesis generales y precisas que se hayan cotejado sistemáticamente con las realidades comprobadas de la política electoral...". Y es que -para el citado autor- el Derecho electoral parece presentar tres tipos importantes de deficiencias: la falta de precisión en la definición de categorías de análisis, la falta de tratamiento sistemático de los datos y la ausencia de explicitación de las reglas de verificación.

En cuanto a la primera de las deficiencias citadas -la falta de precisión terminológica-, y aún teniendo presente el tiempo transcurrido desde la realización de estas afirmaciones, comenzaremos, al igual que la mayoría de los autores que han estudiado cualquiera de las materias de tipo electoral, en este caso la barrera electoral, intentando precisar el contenido de conceptos imprescindibles como "Sistema electoral" y "Derecho electoral". 
Así, por ejemplo, para López AguiLAR (1997), «En ciencia política, ..., la noción instrumental de sistema electoral comprende la totalidad de los elementos interrelacionados en su configuración normativa (los elementos legales y reglamentarios), institucional (administración electoral y garantías) y política (desde la observación de las variables politológicas y socioculturales en las que operan las anteriores). Y ello sin descuidar, además, las relaciones existentes entre este sistema electoral y el propio sistema político, toda vez que las variables normativas del sistema electoral repercuten en la conformación de una dinámica política de características asociadas a aquél... y viceversan. Sistemas que se califican por el resultado y las finalidades que persiguen en mayoritarios $y$ proporcionales.

Precisamente uno de los elementos instrumentales que "completan" habitualmente los sistemas calificados de proporcionales, es el establecimiento de una barrera legal mínima, "Sperrklausel" o "soglia mínima di esclusione" (clausola di sbarramento), que en España no se prevé constitucionalmente pero sí en el desarrollo legislativo posterior tanto por la Ley Orgánica del Régimen Electoral General (LOREG) como en las respectivas regulaciones autonómicas, y que se insertan en él con la finalidad de modular la proporcionalidad. Modulación que, como es sabido, no sólo depende de la existencia de barreras electorales, sino de un conjunto de variables, entre las cuales el tamaño de las circunscripciones electorales juega un papel fundamental.

A su vez, todo el sistema electoral está regulado jurídicamente por lo que se denomina Derecho electoral, disciplina jurídica englobada dentro del Derecho Constitucional, y que según NoHLEN (1977) comprende tanto el conjunto de normas jurídicas que regulan la elección de los órganos representativos, como el conjunto de normas jurídicas que afectan al derecho del individuo a participar en la designación de esos órganos representativos. Estamos, por otro lado, en presencia de una disciplina científica que se encuentra informada por una serie de principios, que configuran una especie de axiológica electoral, y, entre los cuales podemos mencionar los siguientes: el impedimento del falseamiento de la voluntad popular, la conservación del acto electoral y el principio de unidad del acto electoral (ÁLVAREZ ConDE, 1991). Todo ello, como ha puesto de maniesto MASCLET (1989), porque «los caracteres originales del Derecho electoral se explican y justifican por su función, que consiste en respetar el principio democrático".

Por ello, y por lo que se refiere al tema objeto de nuestro estudio, la barrera electoral como elemento configurador de nuestro sistema, 
se deberá tener en cuenta no sólo su regulación legal, sino además la relación con aquellos principios que inspiran el Derecho electoral. Ello es especialmente cierto por lo que al principio democrático se refiere, base del mismo, y en concreto a su manifiestacion más importante, cual es el impedimento del falseamiento de la voluntad popular, que pueden verse afectados por su introducción en él, debiendo respetar los principios de igualdad, proporcionalidad y razonabilidad, principios que deben presidir nuestro modelo de manera que no se desfigure en lo esencial el sistema diseñado constitucionalmente.

Y es que todos los sistemas electorales, quizás en mayor medida los calificados de proporcionales como el nuestro, son susceptibles de modulación a través de determinadas técnicas como los umbrales electorales. Así pues, y como reconoce el propio Tribunal Constitucional, «Es,..., bien conocido que no es posible hablar, sin mayor precisión, de "un" sistema de escrutinio proporcional como algo perfectamente delimitable, de manera univoca, en todos sus contornos, pues todo lo más que puede apreciarse, en la simple afirmación de tal sistema, es la voluntad de procurar, en esencia, una cierta adecuación entre votos recibidos y obtención de escaños». Es más, RAE distingue entre sistema electoral mayoritario y proporcional "de gran magnitud" o "de baja magnitud" y NOHLEN entre "sistemas mayoritarios" y "proporcionales integrales, corregidos $y$ reforzados".

De hecho, son muchos los autores que dudan de la proporcionalidad real de nuestro sistema electoral. Así, por ejemplo, SoLOZÁBAL (1994) califica nuestro sistema, upor lo menos en el terreno de la práctica... como sistema mayoritario atenuado". Para LóPEZ GARRIDo (1994), el sistema electoral español «es proporcional teóricamente y mayoritario en la práctica, que es la peor de las fórmulas». PUNSET (1993) escribe que "es difícil afirmar la observación del principio constitucional que establece "criterios de representación proporcional" para las elecciones" y VALLÉS (1986) precisa que "calificar mecánicamente a nuestro sistema electoral (español) como proporcional responde probablemente a un predominio acrítico de una perspectiva pseudoconstitucionalista o a una comodidad simplificadora, pero no nos ofrece una visión ajustada... A pesar de recurrir a técnicas de carácter proporcional, el vigente sistema electoral español... se inspira en el principio de representación decisión, que lo aproxima -lo confunde-con los modelos conocidos como mayoritarios". Pues, tal y como explican VALLÉs y BosCH, (1997) «la lógica que inspira el sistema se impone sobre la literalidad de la afirmación constitucional». En análogo sentido se pronuncia la generalidad de la doctrina (cfr. por todos MONTERO, LleRA, TORCAL, 1992). 
Se trata de dilucidar, en todo caso, como afecta a nuestro sistema electoral la inclusión de este tipo de barreras. Es decir, si un sistema mayoritario atenuado -expresión utilizada por SÁNCHEZ NAVARRO (1988)- es tan constitucional como un sistema proporcional relativizado, en cuyo caso habrá de procederse a la delimitación. Pensemos en ese sentido, que no cabe identificar sistema proporcional con método de escrutinio o método decisorio y que por tanto son varios los elementos que resultan implicados en la definición y distintos, también, por tanto, los diferentes contenidos y posibilidades que se pueden ofrecer en un sistema calificado de proporcional. No obstante, y como ya ha quedado expuesto, no todos los sectores doctrinales están de acuerdo con está afirmación, pues es cierto que una extralimitación podría no adecuar la denominación a su contenido real.

Según el Tribunal Constitucional, en su Sentencia 40/1981, de 18 de diciembre, representación proporcional es "la que persigue atribuir a cada partido o a cada grupo de opinión un número de mandatos en relación a su fuerza numérica. Cualesquiera que sean sus modalidades concretas, su idea fundamental es la de asegurar a cada partido político o grupo de opinión una representación, sino matemática, cuando menos sensiblemente ajustada a su importancia real."

Es decir, para el Tribunal Constitucional, según sus propias palabras, "La proporcionalidad es, más bien, una orientación o criterio tendencial, porque siempre, mediante su puesta en práctica, quedará modulada o corregida por múltiples factores del sistema electoral, hasta el punto que puede afirmarse que cualquier concreción o desarrollo normativo del criterio, para hacer viable su aplicación, implica necesariamente un recorte respecto a esa "pureza" de proporcionalidad abstractamente considerada" (STC 75/1985, FJ 4. ${ }^{\circ}$.

\section{LA BARRERA ELECTORAL}

La finalidad de las llamadas barreras electorales establecidas en algunos sistemas electorales, es excluir del reparto de escaños a los partidos que no alcanzaran el mínimo de votos fijado por él. Se puede afirmar, por tanto, que cumplen "una finalidad directamente desproporcionalizadora del resultado global de la elección" (VALLÉS Y BOSCH). Es decir, a través de la "barrera mínima», "cláusula de exclusión» o "umbral electoral», los partidos que no obtuvieran ese mínimo de votos necesarios no participarán en el reparto de escaños, evitando de 
esta manera fragmentar en exceso la composición de la Cámara de representación y así coadyuvar en la estabilidad de los gobiernos.

A la postre, lo que se busca con este tipo de técnicas es corregir las fórmulas, que no son sino procedimientos "de cálculo aplicado a los votos expresados por los electores de un distrito con objeto de obtener una distribución de escaños entre los diversos candidatos contendientes" (VALLÉS Y BosCH, 1997), en este caso proporcionales, de tal manera que se atenúe la proporcionalidad de las mismas y así conseguir con ello el objetivo de sistemas, como el mayoritario, que tienen como propósito principal facilitar la estabilidad del gobierno a costa de la no entrada de minorías en el Parlamento que pudieran fragmentarlo en exceso. Los intereses prioritarios perseguidos por los dos sistemas electorales -mayoritario y proporcional- en estado puro son, pues, distintos: la gobernabilidad y la participación de las minorías.

De hecho, las barreras electorales, aparecen con la implantación de los sistemas proporcionales a mediados del siglo XIX, aunque no empezaron a ser utilizados en la generalidad de los países democráticos, hasta principios del siguiente (RIOs RULL, 1997).

No obstante la preocupación que existe respecto de este tipo de umbrales es que su inclusión pueda llegar a desfigurar el sistema calificado formalmente de proporcional. Es decir, el problema se plantea como una cuestión de delimitación y, por tanto, conceptual ya que aunque el sistema se califique de proporcional, a través de estas técnicas correctoras puede transformarse, aunque semánticamente no se presente así, en un sistema mayoritario.

Por ejemplo, el propio Tribunal Constitucional Federal -pensemos al respecto, que ha sido precisamente el Derecho alemán nuestro precedente en esta materia, al que se ha calificado por la doctrina de "modelo paradigmático a este respecto" (FERNÁNDEZ SEGADo, 1986), ya que su legislación prevé la necesidad de que sus candidaturas superen el porcentaje del cinco por cien para poder acceder al reparto electoral, siendo lo más "decisivo" - a juicio del citado autor- la circunscripción en la que se aplica, pues computa a nivel nacional lo que le convierte en "realmente operativo"-como consecuencia de su pronunciamiento en una serie de casos sobre el tema de la barrera electoral, siempre ha expresado su validez considerándola como una garantía legítima de la eficacia de las instituciones parlamentarias, en cuanto tiende a corregir fragmentaciones excesivas en la representación política obtenida mediante la proporcionalidad electoral. (BACHOFF, 1980, Nicolas MUÑIZ, 1977). Y nuestro Tribunal Constitucional, en la misma línea, señala que 
la finalidad de este tipo de reglas es uprocurar combinando incentivos y límites, que la proporcionalidad electoral sea compatible con el resultado que la representación de los electores... no sea en exceso fragmentaria, quedando encomendada a formaciones políticas de cierta relevancia" (STC 75/1985, FJ 4.9).

En todo caso, el órgano encargado de precisar, en ultima instancia, la calificación del sistema ante tales técnicas capaces de desvirtuar la proporcionalidad del sistema es el Tribunal Constitucional, que con motivo de estas cuestiones ya ha tenido ocasión de pronunciarse al respecto. $Y$ es que la barrera legal no debe ser analizada de forma aislada sino en conjunto con los demás elementos que configuran el tipo de sistema así como en relación con los principios que le informan. Pues sólo en conjunción con ellos puede ser descifrado de forma real su impacto sobre la proporcionalidad.

\section{Principios informadores: igualdad, proporcionalidad y razonabilidad}

Los principios que deben ser respetados por nuestro sistema electoral, y por tanto afectan, implican y limitan el alcance de los diferentes elementos que integran el mismo, en este caso la barrera electoral, son tres: la igualdad establecida constitucionalmente en el artículo 23.2, la proporcionalidad enunciada por el 68.3 y la razonabilidad, que más que principio es el criterio interpretativo de creación jurisprudencial utilizado por el Tribunal Constitucional para valorar el efecto o transcendencia de su inclusión en el conjunto del sistema.

\section{a) Igualdad}

El artículo 23.2 de la Constitución establece que los ciudadanos "tienen derecho a acceder en condiciones de igualdad a las funciones $y$ cargos públicos representativos" (GARCía RocA, 1998). Precisamente y como consecuencia del contenido de este precepto, el Tribunal Constitucional ha tenido que decidir algunos recursos de amparo que se presentaron ante él planteando la posibilidad de que la barrera legal vulnerara el artículo señalado, ya que a juicio de los demandantes el derecho ejercido, en este caso el sufragio pasivo, se debe ejercer en condiciones de igualdad, "exigencia en la que no cabe ver sino una concreción del principio que, con carácter general, se reconoce en el 
artículo 14 de nuestra Constitución" (STC 75/1985, de 21 de junio, FJ 4. $\%$. Sobre el principio de igualdad en materia electoral el Tribunal Constitucional ha fijado fundamentalmente dos cuestiones:

1. Que "el principio democrático de la igualdad se encuentra abierto a las fórmulas electorales más diversas, y ello porque se trata de una igualdad ante la Ley, o como el mismo artículo 23.2 de la Constitución establece, de una igualdad referida a las "condiciones" legales en que el conjunto de un proceso electoral se desarrolla, por lo que la igualdad, por lo tanto, no prefigura un sistema electoral y excluye otros, sino que ha de verificarse dentro del sistema electoral que sea libremente determinado por el legislador, impidiendo las diferencias discriminatorias, pero a partir de las reglas de tal sistema, y no por referencia a cualquier otro" (STC 75/1985, de 21 de junio, FJ 4.․).

Es decir, "Lo significativo, ..., es que la regla legal se aplica a todas las candidaturas por igual, sin que conste la exigencia de obstáculos para que todas ellas concurran a unas mismas elecciones, y en unos mismos distritos o circunscripciones en las mismas condiciones legales y sin que conste, tampoco, la existencia de diferencias injustificadas o irrazonables en la aplicación de esa concreta regla, que es por su intrinseca naturaleza enteramente justificada y fundada..." (STC 75/1985, de 21 de junio, FJ 4.ํ).

2. Que no puede identificarse trato igualatorio y criterio electoral de proporcionalidad (SÁNCHEZ NAVARRO 1988 y LÁZARO RIOL 2000), destacando que la ausencia de proporcionalidad, no supondría, por tanto, una vulneración del derecho reconocido en el artículo 23.2 CE (STC 75/1985, FJ 4.ำ y STC 193/1989, FJ 6.․). Es decir, ha dejado claro que igualdad y proporcionalidad del sistema electoral no son lo mismo.

Es necesario concretar, a la vista de lo expuesto por el Tribunal, lo que, a nuestro parecer, son dos vertientes distintas de la actuación del principio de igualdad en el sistema electoral y cuál es su relación con el principio de proporcionalidad. Podriamos decir que la igualdad se relaciona y funciona con respecto al sistema electoral de dos formas: «ad extra" y "ad intra". "Ad extra" por que su relación con el principio de igualdad sólo afecta a la aplicación de la Ley, una aplicación que no debe ser discriminatoria; $y$ "ad intra", porque, una vez que la aplicación se produce en igualdad, puede ocurrir que el contenido de la regulación del proceso electoral sea lo que provoque la discriminación. En este caso, puede ocurrir que la barrera electoral sea tan desproporcionada que ocasione desigualdad en el acceso al reparto de escaños. Es cierto que en los sistemas electorales proporcionales desigualdad de 
reparto siempre existe, pero precisamente por ello debe estar limitada por el principio de proporcionalidad

En puridad, y así lo ha entendido el Tribunal Constitucional, esta vulneración que hemos denominado "ad intra" no proviene de la conculcación del principio de igualdad, sino de otro que aún no contando con el mismo contenido se encuentra íntimamente relacionado con él: el de proporcionalidad. No obstante, lo cierto es que si ambos principios, aun siendo diferentes, no pudieran relacionarse, sería imposible el planteamiento del recurso de amparo como procedimiento de acceso de los particulares al control de la legislación electoral con motivo de la vulneración del art. 68.3 CE.

$Y$ es que aun respondiendo a naturalezas y contenidos distintos tanto la igualdad (art. 14 y $23.2 \mathrm{CE}$ ) como la proporcionalidad (art. 68.3) son principios que deben ser respetados conjuntamente, para que cualquiera que sea la modulación ésta siempre obedezca a fines u objetivos legítimos y razonables, valorados y controlados en cualquier caso por el Tribunal Constitucional. Por consiguiente es necesario facilitar en la medida de lo posible el acceso a su control.

\section{b) Proporcionalidad}

El artículo 68. 3 de la Constitución establece que «La elección se verificará en cada circunscripción atendiendo a criterios de representación proporcional».

El Tribunal Constitucional ha formulado una interpretación flexible de lo que para él significa un sistema proporcional, afirmando en este sentido que no es necesario que se sigan «unos criterios estrictamente proporcionales", aceptando la "introducción de correcciones o modulaciones al principio de proporcionalidad a la vista de las necesidades derivadas del principio de efectividad en la organización y actuación de los poderes públicos... en tanto... se funde en fines u objetivos legítimos y razonables... y no cause discriminaciones entre las opciones en presencia" (STC 75/1985, FJ 5. y 193/1989, FJ 6.ㄱ), ya que, para él, «es notorio que una proporcionalidad estricta es algo difícil de alcanzar en toda representación, y tanto más cuanto más reducido sea el número de representantes a elegir o el colegio a designar (STC 40/1981, FJ 3.ํ y 36/1990, FJ 2.ํ). Así como, una adecuada representación proporcional sólo puede ser, por definición imperfecta y resultar exigible dentro de un razonable margen de flexibilidad, siempre $y$ 
cuando no llegue a alterarse su propia esencia (STC 40/1981 FJ 1..; 32/1985, FJ 3.ำ 36/1990, FJ 2.\%).

En consecuencia, la proporcionalidad o las desviaciones de la misma enjuiciables en amparo por devenir constitutivas de una discriminación vedada por el art. 23.2 de la Constitución, no pueden ser entendidas de una forma estrictamente matemática, sino que deben venir anudadas a una situación notablemente desventajosa y a la ausencia de todo criterio objetivo o razonamiento que las justifique (SSTC 75/1985, FJ 3.; 36/1990, FJ 2.ำ 4/1992, FJ 2.)" (STC 30/1993, de 25 de enero, FJ 7.ㅇ).

$Y$ es que es jurisprudencia reiterada del Tribunal que «la exigencia de proporcionalidad ha de verse como un imperativo de "tendencia" que orienta, pero no prefigura, la libertad de configuración del legislador democrático en este ámbito (SSTC 40/1981, 75/1985, 193/1989 y 45/19992, entre otras)" (STC 225/1998, de 23 de noviembre, FJ 7.-). O lo que es lo mismo, «la representación proporcional es la que persigue atribuir a cada partido o a cada grupo de opinión un número de mandatos en relación con su fuerza numérica. Cualesquiera que sean sus modalidades concretas, su idea fundamental es la de asegurar a cada partido político o grupo de opinión una representación si no matemática, cuando menos sensiblemente ajustada a su importancia real" (STC 40/1981, de 18 de diciembre, FJ 2.\%).

No obstante, y aún partiendo de una interpretación flexible del contenido de la proporcionalidad, la importancia real de su afirmación radica en la aplicación que de ella haga el Tribunal ante situaciones concretas como, en este caso, la valoración de la inclusión de barreras electorales $y$, más particularmente, en cuanto al porcentaje y modalidades de las mismas.

\section{c) Razonabilidad}

Ante la falta de precisión de los elementos y características que definen un sistema proporcional, así como de la graduación de los mismos, y en concreto del umbral electoral, el Tribunal Constitucional ha tenido que decidir en cada uno de los casos de forma particular y recurriendo a lo que se ha denominado "test de razonabilidad", para poder concretar si determinadas técnicas como la analizada, desfiguran de tal manera el sistema que no podría ser calificado de proporcional.

Es decir, ha tenido que analizar de forma singularizada en cada uno de los recursos presentados ante él, si la desigualdad que provoca 
la diferencia de trato entre candidaturas a la hora de acceder a la atribución de escaños no se encontraba desprovista de una justificación objetiva y razonable como "la necesidad de asegurar, según el legislador autonómico, una suficiente presencia de las fuerzas políticas parlamentarias en el ámbito de toda la región" (STC 193/1987, de 16 de noviembre, FJ 4.. ), que la amparase.

Por ejemplo, el Tribunal Constitucional en la STC 72/1989, de 20 de abril, y como consecuencia de la posibilidad de inconstitucionalidad de la barrera electoral canaria del 20 por 100 de los votos válidamente emitidos en la circunscripción electoral, expresó lo siguiente:

"no resulta inconveniente dejar aquí constancia de que la regla del porcentaje mínimo del 20 por 100 de los votos emitidos en la circunscripción electoral, establecida en el artículo 8.2 del Estatuto Canario, no merece, en modo alguno, las calificaciones de exhorbitante o contrario al sistema electoral de representación proporcional, sino muy claramente la de plenamente razonable y adecuada a las peculiaridades de su régimen electoral, puesto que, organizado éste sobre las circunscripciones de las Islas de Hierro, Fuerteventura, Gran Canaria, La Gomera, Lanzarote, La Palma y Tenerife -art. 8.4 del Estatuto-, con la indudable finalidad de conformar el Parlamento con representaciones políticas de los ciudadanos de cada una de esas islas, dicho porcentaje del 20 por 100 insular asegura tal finalidad legal al actuar como correctivo del 3 por 100 regional, ya que no de estar así previsto alguna de dichas circunscripciones electorales no podrían alcanzar representación parlamentaria, dado que su número de votantes, e incluso electores, no es suficiente para superar el 3 por 100 de los votos válidos emitidos en la Región..."

También tuvo ocasión de realizar dicho test como consecuencia de la duda de constitucionalidad que se suscitó a raíz del planteamiento de un recurso de amparo con respecto al artículo 15 de la Ley 2/1987, de Elecciones a la Asamblea Regional de Murcia. Pero en este caso con respecto a una barrera del 5 por 100 de los votos validamente emitidos y además aplicándose el porcentaje mínimo no a cada circunscripción sino a toda la Región:

«En el caso presente, la lectura del artículo 15 a) de la Ley 2/1987 revela la pretensión del legislador autonómico de primar a aquellos partidos, coaliciones o agrupaciones de electores que tengan suficiente presencia o aceptación electoral en el conjunto de la región y en detrimento de aquellas otras circunscritas a un ámbito local más reducido y evitando asi una mayor fragmentación parlamentaria por razones de carácter geo- 
gráfico o territorial. Pues bien, esta finalidad, querida por el legislador, no puede tacharse de irrazonable o arbitraria, ya que el propio art. 21.1 del Estatuto de Murcia prescribe que la Asamblea Regional representa "al pueblo de la Región de Murcia", pero no prescribe (aunque tampoco lo prohibe) una representación estrictamente proporcional de las concretas circunscripciones, que son meros instrumentos o demarcaciones del procedimiento electoral a la hora de traducir en un concreto número de representantes los sufragios válidamente emitidos; por lo que la fórmula elegida por el legislador constituye sin duda una opción legítima, aunque evidentemente, no la única legítimamente posible." (FJ 4.9)

Ese mismo estudio de razonabilidad o valoración conjunta de las cláusulas o modulaciones del sistema, es el que siguió el Tribunal Constitucional cuando se planteó recurso de inconstitucionalidad contra el apartado segundo de la Disposición Transitoria Primera de la Ley Orgánica 4/1996, de 30 de diciembre, de reforma de la Ley Orgánica 10/1982, de 10 de agosto del Estatuto de Autonomía de Canarias, que establece "que sólo serán tenidas en cuenta aquellas listas de partido coalición que hubieran obtenido el mayor número de votos válidos de su respectiva circunscripción electoral y las siguientes que hubieran obtenido, al menos el 30 por 100 de los votos válidos emitidos en la circunscripción insular o, sumando los de todas las circunscripciones en donde hubiera presentado candidatura, al menos el 6 por 100 de los votos válidos emitidos en la totalidad de la Comunidad".

Fue el Defensor del Pueblo el que contra el citado precepto interpuso recurso de inconstitucionalidad por estimar "que vulnera el artículo 9 del propio Estatuto de Autonomía (integrante del bloque de constitucionalidad) así como los artículos 1.1, 6, 9.2, 14, 23.2 y 152.1" del texto constitucional, ya que a su juicio el resultado del sistema que se introduce hace "que el carácter proporcional más que modulado quede completamente eliminado, al obstaculizar las posibilidades de representación de minorias que pueden ser mayoritarias".

El Defensor, siguiendo su "test de razonablidad", es decir analizando si las modificaciones introducidas cuentan o carecen de un criterio objetivo y razonable que pueda justificarlas, llega a conclusiones distintas de las del Alto Tribunal. Porque si bien es cierto, como ya hemos expuesto, que el sistema proporcional puede presentarse con diferentes modulaciones o variantes, no lo es menos el que el exceso puede provocar el falseamiento del sistema que denominado como proporcional, en realidad por su contenido y consecuencias no se comporta como tal. 
En ese sentido el Defensor del Pueblo en su Dictamen se plantea, por ejemplo, "si la referencia a la lista que hubiera obtenido el mayor número de votos válidos de su respectiva circunscripción, ..., no supone una violación del sistema proporcional..." o "¿los límites o topes del $6 \%$ en toda la Comunidad Autónoma y el $30 \%$ en cada circunscripción, no causan discriminación entre las opciones en presencia, no dan lugar a una situación notablemente desventajosa y responden a un fin legitimo y razonable? Pensemos al respecto en una de las cuestiones analizadas por el Defensor a modo de ejemplo y como apoyo a su argumentación, y que pone de relieve el distinto resultado del estudio:

"la supuesta alternancia de ambas barreras sólo puede darse en las dos islas centrales, en las que se ha solido recurrir preferentemente a la barrera regional, mientras que las candidaturas insulares únicamente han podido operar con la otra barrera electoral. Con ello, se produce una mayor discriminación de las candidaturas de las islas menores frente a las candidaturas de las islas centrales, pues necesitan superar un mayor número de votos que aquellas para superar la barrera, obligándolas, con ello, a formar parte de coaliciones suprainsulares, que muchas veces presentan un carácter artificial, influyendo, a la postre, en la inestabilidad gubernamental de la Comunidad Autónoma, y en la inevitable sospecha del agravio comparativo».

Añadiendo, además, lo discriminatorio del sistema por

"el hecho de que podría producir que dos partidos con idéntico resultado en dos islas diferentes con en el mismo número de escaños obtuvieran escaño o no en función de haber sido o no la lista más votada" o, también, como "la elevación del $3 \%$ al $6 \%$ hace que ni siquiera todo el censo electoral de Fuerteventura sirva para superar la barrera regional $(6 \%)$, y que incluso los censos considerados conjuntamente de Lanzarote y La Palma la superen por muy poco (ya el $3 \%$ anterior no era superado por el censo electoral de las islas de El Hierro y La Gomera)".

Pues bien, las barreras impuestas por el citado precepto son analizadas en conjunto por la STC 225/1998, de 23 de noviembre (FJ 7.9), en la que el Tribunal Constitucional, teniendo en cuenta una serie de cuestiones o piezas claves del sistema electoral canario que la propia Sentencia presenta, concluye con la imposibilidad de afirmar que el sistema de cláusulas presentado sea contraria a la proporcionalidad exigida. En este sentido afirma que: 
1. La configuración de este tipo de barreras operan como criterio parcialmente corrector de la proporcionalidad y encuentran apoyo en el denominado hecho insular.

2. Su finalidad no es exclusivamente restringir los efectos proporcionales de la fórmula electoral utilizada, sino evitar la excesiva fragmentación política de la Cámara favoreciendo la estabilidad gubernamental.

3. La "barrera regional" (6 por 100) favorece a las fuerzas políticas mayoritarias. Lo que supone favorecer la estabilidad gubernamental.

4. El fin perseguido de la "barrera local o de la circunscripción» (30 por 100) es el de asegurar la presencia de fuerzas políticas mayoritarias en la circunscripción, pero minoritarias en el conjunto de la Comunidad Autónoma. Por ello, supone un correctivo de la primera en beneficio de la representación de cada isla, de forma que permite la presencia en el Parlamento canario de candidaturas de fuerte implantación insular, aunque con escaso nivel de voto en su valoración global desde la óptica del voto emitido en el conjunto de la Comunidad Autónoma.

5. La tercera de las reglas, la que posibilita el acceso a la distribución de escaños a la candidatura que hubiera obtenido uel mayor número de votos válidos en la circunscripción", "tampoco puede ser -a juicio del Tribunal- considerada aisladamente... su conexión sistemática con las otras cláusulas de exclusión, pone de relieve que, en puridad, opera como una corrección de las antedichas barreras, permitiendo alcanzar representación parlamentaria a la candidatura más votada en una circunscripción, aunque no alcanzase el 30 por 100 del voto dentro de la misma, ni el 6 por 100 en el conjunto de la Comunidad Autónoman.

No obstante, $y$ en contra, se han pronunciado también algunos sectores doctrinales que no coinciden con el análisis y conclusiones de la STC 225/1998. Este es el caso de LóPEz AGUILAR cuando afirma, por ejemplo, que "En la práctica, la "vuelta de tuerca" así operada trasluce tanto como evidencia una intención política cuya constitucionalidad merece ser discutida: congelar -petrificar, si se quiere- el actual espectro parlamentario canario, haciendo casi inevitable cualquier "inmisión" de ninguna fuerza insularista que no desee coligarse o confederarse en CC ("advenedizos abstenerse")". Añadiendo, además, que «con la nueva redacción no solamente se potencian las trabas irrazonables al acceso al cargo público representativo en condiciones de 
igualdad (art. 23.2 CE), sino que se crea un nuevo frente de desigualdad carente de parámetro alguno de objetivización: ser "la fuerza más votada" puede ser, en la práctica y la experiencia electoral, resultante aleatoria de la distribución y/o dispersión del voto de cada circunscripción insular..., con total independencia del volumen de población al que cuantitativamente se represente, siendo o no siendo, en cada caso, "el más votado" en la isla». O YANES HerRero (1997) que ya la barrera legal del 20 por 100 era por él calificada de uinusitada».

Sin embargo, para Fernández Segado, este tipo de fórmulas no vulneran el principio de igualdad pues ya en 1986, proponía, aunque fuera para las Elecciones Generales, "la fórmula del 3 por 100 de los votos a nivel nacional con carácter generalizado y, a título de excepción, un porcentaje que podría oscilar entre el 10 y el 15 por 100 de los votos, computando a nivel autonómico o, simplemente, a nivel del conjunto de circunscripciones en que presentaran candidaturas, fórmula que regiría para aquellos partidos o coaliciones que se presentaran tan sólo en un ámbito territorial previamente delimitado; en definitiva para las formaciones de carácter regional o, si se prefiere, nacionalistas". En parecidos términos ya se había pronunciado MARTínez CuADRADo (1981). Frente a estas tesis, nosotros no nos mostramos partidarios de la existencia de estas cláusulas tope. Y ello, porque las diversas técnicas electorales no pueden ni deben condicionar, aunque su incidencia no sea especialmente relevante, la presencia parlamentaria de las diferentes opciones políticas, la cual únicamente debe venir determinada por el apoyo popular de que disponen.

Como es evidente, la interpretación y, por ende, la solución del denominado "test de razonabilidad" es variable en tanto en cuanto en él influyen no sólo el comportamiento de los distintos elementos que configuran el Sistema electoral sino además las diferentes relaciones que pueden mantener entre si. De ahí que las conclusiones puedan ser varias, aunque la única determinante y definitiva sea la del Tribunal Constitucional.

\section{Relación con los demás elementos que configuran el sistema electoral}

Al igual que el estudio de los principios que informan nuestro sistema electoral, con respecto a la barrera electoral también es necesario hacer un seguimiento de su relación con los demás elementos configuradores del mismo. De hecho, VALLÉS y BOSCH incluyen la "barrera 
mínima" en lo que han denominado como variable independiente del sistema electoral a raíz de la distinción que ellos mismos proponen entre la perspectiva desde las que parecen ser necesarias el estudio del Derecho electoral: perspectiva politológica y la perspectiva jurídica (es en esta última donde se incluye la barrera electoral como elemento del sistema).

Su relación con las demás variables, tal y como ha destacado la generalidad de la doctrina, será imprescindible a la hora de delimitar su impacto efectivo en el sistema electoral. Según Rios RULL (1997), "son dos los elementos de especial importancia para poder abordar el objeto de nuestro estudio desde una perspectiva jurídico-positiva: la circunscripción y las candidaturas; ambos conceptos unidos al modo de escrutinio nos van a dar la clave de las barreras electorales». Para este autor, por tanto, son tres los elementos configuradores del sistema electoral: voto, circunscripción y fórmula electoral. Nosotros, además, añadiríamos un dato importante con respecto al tema que nos ocupa. Un aspecto relacionado con la circunscripción pero que creemos tiene entidad propia en este caso como para ser también señalado individualmente: la magnitud o número de escaños a repartir en cada circunscripción.

Así pues, del análisis de la barrera electoral desde su propia naturaleza y desde su relación con el resto de los elementos integrantes del sistema, caben destacar las siguientes conclusiones:

1. La barrera electoral mínima se puede fijar bien en todo el territorio en el que se produce la elección, a nivel de distrito o circunscripción o combinando ambas.

2. Parece que cuanto más elevada es la barrera menor será el número de partidos que tendrán acceso al arco parlamentario y, por tanto, la fragmentación será menor contribuyendo con ello a la estabilidad de los Gobiernos. De hecho, y como así se ha señalado por la doctrina (VALLÉS Y BOSCH) "Un análisis empírico comparado señala que el efecto reductor de la fragmentación es perceptible a partir de una barrera efectiva del 6 por ciento. Así, cuando la barrera es menor al 6 por ciento, la media de partidos electorales es de 4,3 y la de partidos con representación parlamentaria es de 3,9. Cuando la barrera se sitúa entre el 8 y el 12 por ciento, la medida de partidos electorales se sitúa en torno a 4 y la de partidos parlamentarios en torno a 3,3".

3. Tal y como explican VALLES Y BOSCH, "El apoyo electoral mínimo exigido para la participación en el reparto de escaños puede 
expresarse en votos o escaños. En el primer caso, se establece legalmente el porcentaje de votos indispensable para intervenir en la distribución de puestos. Alternativamente, puede fijarse una cantidad absoluta de votos -en lugar de un porcentaje-como medida de apoyo electoral mínimo". Así, "La barrera puede expresarse también en escaños cuando la asignación de los mismos se hace en un doble nivel de circunscripción. En este supuesto, sólo se admite el reparto en el segundo nivel -estatal o regional- a los partidos o grupos que hayan obtenido algún o algunos escaños en el primer nivel de competición". El ejemplo más conocido, al que hemos aludido en apartados anteriores, es el del sistema electoral alemán, que para acceder a la distribución de escaños a escala federal establece una barrera mínima expresada alternativamente en el 5 por ciento del total de los votos federales o bien aquellos que hubiesen obtenido en la elección un mínimo de tres escaños directos en las circunscripciones uninominales por mayoría relativa.

4. Las barreras a escala general perjudican a los partidos minoritarios. Así, "una barrera mínima a escala estatal perjudica a los partidos que pueden tener presencia concentrada en pocos distritos. Es el caso de los partidos nacionalistas o regionalistas, implantados únicamente en una parte del territorio estatal (VALLÉS Y BOSCH). En cambio, una barrera mínima en cada distrito perjudica a los partidos menores que tienen un apoyo disperso, repartido de manera relativamente equilibrada a lo largo del país. Desde esta perspectiva, la introducción de barreras mínimas presenta problemas de legitimidad del sistema..." (VALLÉS Y BOSCH).

5. Los efectos de la barrera electoral en sistemas con distribución de escaños en más de un nivel -o combinación de circunscripciones- afectarán de modo diferente a la proporcionalidad según sea el tipo de combinación que se establezca. Así, «cuando la combinación se basa en la transferencia de restos de un nivel a otro, la fijación de barreras elevadas en el segundo nivel -más amplio- de distribución de escaños perjudica a los partidos pequeños. Por ello, los partidos mayores prefieren que la cuota fijada para el primer nivel sea suficientemente alta para aumentar los escaños disponibles en el segundo reparto -al que ya no acceden los partidos menores, excluidos por el efecto de la barrera" (VALLÉS Y BoSCH).

6. Son varias las posibles combinaciones para la fijación de barreras tal y como ha señalado la doctrina. Junto a ella, la circunscripción y, especialmente, la magnitud son los elementos que más pueden llegar a condicionar el resultado. Por esta razón, la decisión política que 
las promueve tiene en cuenta dichos elementos a la hora de distribuir los apoyos electorales a los partidos mayoritarios o minoritarios. Es más, NOHLEN advierte, por ejemplo, que «los cambios en los tamaños de las circunscripciones electorales pueden implicar... un cambio del sistema electoral».

Así, la magnitud, es decir, el número de escaños que deben ser designados por los electores de la circunscripción correspondiente (en España, en la elección al Congreso de los Diputados la magnitud es media 6,73 escaños, vid. sobre el tamaño del distrito RAE, cuando la gama oscila entre el 5 por 100 de los votos en Alemania y 0,67 por 100 estatal de la barrera holandesa), es uno de los elementos claves en el mantenimiento de la proporcionalidad de un sistema electoral que puede dejar sin fundamento la introducción de la barrera legal.

7. La aplicación de la barrera a distritos de baja magnitud tiene escaso impacto, porque el porcentaje de votos efectivamente necesario para conseguir uno de los pocos escaños en disputa no depende, en la mayoría de los casos, de la barrera. La barrera puede ser efectiva en circunscripciones de alta magnitud, donde un porcentaje menor de votos podría dar opción a conseguir un escaño (Vid. Nohlen, CaRRERAS Y VALLÉS, VALLÉS Y BOSCH).

Es decir, cuanto mayor es la magnitud de la circunscripción, es decir, cuanto mayor es el número de escaños a repartir en la circunscripción las barreras tienen más "impacto" o "despliegan mayor eficacia” (Bravo de Laguna, 1996), llegando a la conclusión -como Yanes Herreros- de que su función es uornamental", cuyo resultado responde más bien a una «reproducción mimética e irreflexiva de una moda que en una fundada preocupación por evitar la excesiva fragmentación parlamentaria".

Y es que, como argumenta Bravo de LAGUNA, ula magnitud de las circunscripciones está directamente relacionada con la proporcionalidad electoral, de modo que en las circunscripciones pequeñas la elección es siempre mayoritaria, a pesar de que utilicemos fórmulas electorales proporcionales, y sólo a partir de 6 escaños podemos hablar en puridad de elección proporcional en una circunscripción, lo que, por ejemplo, tiene efectos importantes de desproporcionalidad en el sistema electoral general español. La proporcionalidad en una circunscripción aumenta con el número de escaños a elegir dentro de cada fórmula electoral, pero a partir de 20 ya no mejoran. Es decir, la barrera natural del tamaño de la circunscripción afecta mucho más que la barrera legal. 
Sobre el tamaño grande, mediano o pequeño de la circunscripción en función de su magnitud hay varias posturas doctrinales. Asi por ejemplo, para FERNÁNDEZ SEGADO, magnitud baja se corresponde a un número inferior a 6 , y medio baja entre 6 y 10; para VIDAL PRADo (1995), por debajo de 5 escaños se considera pequeña, entre 6 y 9 mediana y de 10 en adelante grande; y Fralle CliviLlés (1997), afirma que en nuestro sistema la cifra que actúa moldeando de una u otra forma la representación es el 7.

\section{LA BARRERA EN EL SISTEMA ELECTORAL ESPAÑOL}

\section{Elecciones generales}

Como es sabido, la barrera electoral en nuestro ordenamiento es un elemento introducido no constitucionalmente sino través de la LOREG. De ahí que muchos autores la denominen "barrera legal». Un elemento que apenas fue debatido en el trámite parlamentario aun cuando los diputados contrarios a la ley defendían su supresión (VIDAL Prado).

El artículo 163.1.a) de la LOREG establece que una de las reglas para la atribución de escaños en función de los resultados será que no se tendrán en cuenta aquellas candidaturas que no hubieran obtenido, al menos, el 3 por 100 de los votos válidos emitidos en la circunscripción.

No obstante, sobre el efecto real de la barrera legal en nuestro sistema, FERNÁNDEZ SEGADO ya opinaba en 1986, al igual que la mayoría de la doctrina posterior, que no creía que dicha cláusula pudiera actuar como elemento corrector del excesivo fraccionamiento parlamentario, calificando su operatividad de "mínima, por no decir de nula" (también en este sentido VALLÉS Y BOSCH Y VIDAL PRADO). Y ello, explicaba, "no ya por no alcanzar tan sólo el umbral del 3 por 100 , sino, esencialmente, porque su cómputo se verifica a nivel de cada circunscripción en particular, que no a nivel nacional». Por tanto, le parece que la cláusula sólo puede ofrecer alguna operatividad en las circunscripciones de tamaño alto: Madrid y Barcelona. Es más, afirmaba que se puede constatar empíricamente que en las tres elecciones generales celebradas hasta 1986 "las listas de partidos que no han sido tenidas en cuenta en el momento de proceder al reparto de escaños por no alcanzar el listón del 3 por 100 de los votos válidos de la circunscripción, no habrían tenido posibilidad alguna de alcanzar representación parlamentaria de no haber existido barrera legal». 
Es decir, y como posteriormente ha sido confirmado, únicamente tendría operatividad real si nuestro sistema electoral contara con otras fórmulas electorales o se ampliara el número de diputados (VIDAL Prado). Al respecto Fraile Clivillés explica que analizando la composición de la Cámara de la Legislatura 1996-2000, en todas las provincias en la que el-número de representantes a elegir es $3,4,5$ ó 6 no hay más que representación de dos partidos. Sin embargo, a partir de 7, se incluye al menos uno más. $Y$ es que, tal y como especifica Fernández SEgADO, 30 de las 52 circunscripciones no alcanzan el tamaño de 6 , umbral necesario para que el sistema se comporte como proporcional. Por ello, para el citado autor la barrera del 3 por 100 cumple más bien un efecto psicológico que realmente disuasorio de minorías.

No obstante es de destacar que como consecuencia de las elecciones a Cortes Generales celebradas el 6 de junio de 1993, el CDS interpuso recurso de amparo contra la decisión de la Sección Séptima de la Sala de lo Contencioso-Administrativo del Tribunal Supremo, que desestimaba el recurso contencioso-electoral 498/1993, interpuesto contra la proclamación de electos al Congreso de Diputados, entre los que no figuraba el candidato núm. 1 de la lista presentada por dicho Partido, al no haber conseguido la mencionada lista superar el 3 por 100 de los votos válidos emitidos en la circunscripción, alegando, además, que se computaron los votos en blanco entre los votos válidos emitidos, tal y como establece el art. 108.4 de la LOREG. En cualquier caso, y como en sentencias anteriores, se desestimo el recurso (STC 265/1993, de 26 de junio).

Parece, pues, que la relación con otros elementos del sistema hace de la barrera un elemento inocuo que en nada afecta a la proporcionalidad del sistema, pues en la mayoría de ocasiones es necesario un porcentaje de votos superior al fijado por la propia barrera. Y es que es necesario distinguir entre barrera legal y barrera efectiva. Por ello, entendemos que la barrera legal se introduce más bien para actuar como pieza-cierre del conjunto en el que se engloban las demás variables que configuran el sistema electoral y que, considerado en su totalidad, es el que en definitiva actúa como verdadera limitación.

\section{Elecciones autonómicas}

La Constitución en materia electoral autonómica únicamente establece en su artículo 152.1 que, en los Estatutos aprobados por el procedimiento del artículo 151, "la organización institucional autonó- 
mica se basará en una Asamblea, elegida por sufragio universal, con arreglo a un sistema de representación proporcional que asegure, además, la representación de las diversas zonas del territorio...". Por tanto, sin entrar en el debate sobre el ámbito de aplicación del artículo, ya que ha sido interpretado de forma amplia por la doctrina, entendiendo incluidas también en él a las Comunidades Autónomas que no accedieron por dicha vía, son las legislaciones respectivas las que van a regular sus sistemas electorales a través de sus Estatutos y Leyes de desarrollo.

Así, todas las regulaciones autonómicas han optado por un sistema proporcional, el modelo de escrutinio D'Hondt y la inclusión en sus sistemas de barreras electorales. Las opciones escogidas en función del tipo de umbral son:

1. Barrera del 3 por 100 con redacciones todas muy similares, en las cuales se establece una cláusula señalando que "no se tendrán en cuenta aquellas candidaturas que no hubieran obtenido, al menos, el tres por cien de los votos válidos emitidos en la circunscripción". En este primer grupo se encontrarían las Comunidades Autonomas de Andalucía (art. 18.1. a) de la Ley 1/1986, de 2 de enero, por la que se regulan las elecciones al Parlamento de Andalucía), Aragón (art. 14 a) de la Ley 2/1987, de 16 de febrero, por la que se regulan las elecciones de Diputados a las Cortes de Aragón), Asturias (art. 13.1. a) de la Ley 14/1986, de 26 de diciembre, de elecciones a la Junta General del Principado), Castilla-La Mancha (art. 19 a) de la Ley $5 / 1986$, de 23 de diciembre, reguladora de las elecciones a Diputados), Castilla-León (art. 20 a) de la Ley 3/1987, de 30 de marzo, de regulacion de elecciones a Cortes), Navarra (art. 10.1. de la Ley Foral 16/1986, de 17 de noviembre, de regulación de las elecciones al Parlamento), País Vasco (art. 11.1 de la Ley 5/1990, de 15 de junio de elecciones al Parlamento Vasco, modificado por Ley $6 / 2000$, de 4 de octubre) y Cataluña, que, como es sabido, carece de una autentica ley electoral.

2. De similar redacción pero fijando el umbral en el 5 por 100. En este segundo grupo se encontrarían las Comunidades Autónomas de Cantabria (art. 17.3. de la Ley 5/1987, de 27 de marzo, de elecciones al. Parlamento de Cantabria), Galicia (art. 10 a) de la Ley 8/1985, de 13 de agosto, de elecciones al Parlamento de Galicia), Madrid (art. 10.6. de la Ley Orgánica 3/1983, de 25 de febrero, por la que se aprueba el Estatuto de Autonomía), Murcia (art. 15 a) de la Ley 2/1987, de 12 de febrero, que regula las elecciones a la Asamblea), La Rioja (art. 20 a) de la Ley 3/1991, 
de 21 de marzo, de elecciones a la Diputación General), Valencia (art. 12.2. de la Ley Orgánica 5/1982, de 1 de julio por la que se aprueba el Estatuto de Autonomia y art. 12 a) de la Ley 1/1987, de 31 de marzo, de elecciones a Diputados de las Cortes Valencianas), Islas Baleares (art. 12.3. de la ley $8 / 1986$, de 26 de noviembre, que regula las elecciones al Parlamento de las Islas Baleares) y Ceuta y Melilla (cuyos Estatutos se remiten al sistema utilizado para las elecciones municipales).

3. $Y$ con barreras alternativas: Canarias que según la Disposición Transitoria 1". 2 de su Estatuto establece que "sólo serán tenidas en cuenta aquellas listas de partido o coalición que hubieran obtenido el mayor número de votos válidos de su respectiva circunscripción electoral y las siguientes que hubieran obtenido, al menos, el 30 por 100 de los votos válidos emitidos en la circunscripción insular 0 , sumando los de todas las circunscripciones en donde hubiera presentado candidatura, el menos, el 6 por 100 de los votos válidos emitidos en la totalidad de la Comunidad" y Extremadura que según el art. 19.1 a) de la Ley 2/1987, de 16 de marzo, de Elecciones a la Asamblea, "Para que una candidatura sea tenida en cuenta deberá obtener, al menos, el cinco por ciento de los votos válidos emitidos en la circunscripción a la que concurra. No obstante, si una candidatura no lograra dicho porcentaje, será tenida en cuenta para atribución de escaños siempre que cumpla las siguientes condiciones:

a) Que el partido, coalición, federación o agrupación de electores al que representa haya presentado candidatura en las dos circunscripciones.

b) Que el total de los votos válidos conseguidos por ambas candidaturas sea igual o superior al cinco por ciento de la suma de los votos válidos emitidos en las dos circunscripciones."

Con la aplicación de estas barreras es cierto que se han dado casos de exclusión de candidaturas en el reparto de escaños, pero no lo es menos que debería hacerse un estudio conjunto con el resto de elementos que influyen en la proporcionalidad del sistema para saber, como hemos venido reiterando, el impacto real de su aplicación. Partiendo de la base de que todas contemplan el sistema proporcional como opción y el modelo de escrutinio D'Hondt, las variantes se producen también por el tipo de circunscripción electoral por la que han optado y la magnitud de las mismas.

Por ejemplo, las Comunidades Autónomas de Cantabria, Madrid y La Rioja son muestra de exclusión de candidaturas en el reparto de 
escaños por efecto de la barrera electoral del 5 por 100 . Al respecto se presentan unos datos proporcionados por Rios RULL, que explica que el umbral «En Cantabria, cuyo límite estaba en 15.152 votos, ha dejado sin representación a IU y CDS, con 13.023 votos $(4,29$ por 100$)$ y 7.926 $(2,61$ por 100$)$ respectivamente, cuando la adjudicación del último escaño se hizo con 6.434 votos (PSOE). En Madrid, con la barrera en 112.068 votos, no tuvo en cuenta a las Candidaturas del CDS y de los Verdes con 74.531 (3,32 por 100) y 35.559 votos $(1,59$ por 100$)$, respectivamente, mientras que el último electo lo fue con 20.340 votos. Por último, La Rioja, cuya Cláusula está fijada en 7.293 votos ha dejado sin acceso a escaño a IU y CDS, con $6.499(4,45$ por 100$)$ y 6.271 votos $(4,29$ por 100), respectivamente, y la última acta de Diputado regional se consiguió con 3.802 votos.»

De lo dicho con anterioridad se desprende que la barrera puede ser más o menos efectiva en su función moduladora de la proporcionalidad según la regulación que se haga de dos importantes variantes: el tipo de circunscripción y su magnitud. Que hacen que cuanto mayor sea la circunscripción y la magnitud de la misma, mayor también será la proporcionalidad.

Así, por ejemplo, es de destacar como en los sistemas electorales de las Comunidades Autónomas, aquellas que prevén la circunscripción única aplican una barrera del 5\% (La Rioja, Murcia, Madrid, Cantabria y Valencia, éstas tres últimas además con un número elevado de escaños), y aquellas en las que la circunscripción no es única y su magnitud, salvo excepciones -por ejemplo Cataluña- también es menor, la barrera se fija en un 3 por 100. Salvo Navarra que siendo de circunscripción única exige un 3 por 100 o Baleares y Galicia que no siéndolo la fijan en un 5 por 100 . No obstante, y en relación con la circunscripción única, cabría preguntarse si su elección y utilización como baremo en la cláusula de exclusión, podría vulnerar de alguna manera el artículo 152.1, párrafo 1., según el cual las Asambleas Legislativas de las Comunidades Autónomas deben ser elegidas asegurando la representación de las diversas zonas del territorio, sobre todo en aquellos casos en los que la circunscripción electoral es modificada a tal efecto como en el único caso, el de la Comunidad Valenciana, en el que, aún previéndose la provincia como circunscripción electoral, la barrera del 5 por 100 se aplica sobre la totalidad de los votos emitidos en la Comunidad (vid. la STC 193/1983 (Antecedente núm. 3)).

Al respecto se presenta un cuadro con los datos más significativos: 


\begin{tabular}{|c|c|c|c|}
\hline $\begin{array}{l}\text { Comunidades } \\
\text { Autónomas }\end{array}$ & Barrera electoral & Circunscripción (núm.) & Magnitud \\
\hline ANDALUCIA & $3 \%$ de la circunscripción & Provincia (8) & 109 \\
\hline ARAGÓN & $3 \%$ de la circunscripción & Provincia (3) & 67 \\
\hline ASTURIAS & $3 \%$ de la circunscripción & Centro/Occidente/Oriente & 45 \\
\hline BALEARES & $5 \%$ de la circunscripción & Islas (4) & 59 \\
\hline CANARIAS & $\begin{array}{l}6 \% \text { de los votos el conjunto } \\
\text { del archipiélago o } 30 \% \text { de } \\
\text { los votos emitidos en la Isla }\end{array}$ & Islas (7) & 60 \\
\hline CANTABRIA & $5 \%$ & Comunidad Autónoma & 39 \\
\hline CATALUÑA & $3 \%$ de la circunscripción & Provincia (4) & 135 \\
\hline $\begin{array}{l}\text { CASTILLA- } \\
\text { LA MANCHA }\end{array}$ & $3 \%$ de la circunscripción & Provincia (5) & 47 \\
\hline $\begin{array}{l}\text { CASTILLA } \\
\text {-LEON }\end{array}$ & $3 \%$ de la circunscripción & Provincia (9) & 84 \\
\hline EXTREMADURA & $\begin{array}{l}5 \% \text { (con excepción) de la } \\
\text { circunscripción }\end{array}$ & Provincia (2) & 65 \\
\hline GALICIA & $5 \%$ de la circunscripción & Provincia (4) & 75 \\
\hline MADRID & $5 \%$ de la circunscripción & Comunidad & 101 \\
\hline MURCIA & $5 \%$ de la Región & 5 demarcaciones electorales & 45 \\
\hline NAVARRA & $3 \%$ de la circunscripción & Territorio Foral & 50 \\
\hline PAIS VASCO & $3 \%$ de la circunscripción & Territorios Históricos (3) & 75 \\
\hline LA RIOJA & $5 \%$ de la circunscripción & Comunidad Autónoma & 33 \\
\hline VALENCIA & $\begin{array}{l}5 \% \text { de la Comunidad } \\
\text { Autónoma }\end{array}$ & Provincia (3) & 89 \\
\hline $\begin{array}{l}\text { CEUTA Y } \\
\text { MELILLA }\end{array}$ & $5 \%$ de la circunscripción & Término Municipal & 25 \\
\hline
\end{tabular}

Aún así, y con independencia de las variables que hayan provocado realmente la exclusión de algún candidato, el Tribunal Constitucional ha conocido cinco asuntos relacionados con la barrera electoral, de los cuáles cuatro lo han sido como consecuencia de elecciones a Parlamentos Autonómicos: Cataluña con un recurso de amparo planteado por la coalición electoral "Entesa de l'Esquerra Catalana" y por el "Partit dels Comunistes de Catalunya" (STC 75/1985)); Canarias: se han interpuesto dos recursos de amparo, el primero por D. Gregorio Toledo en nombre propio y como candidato de la coalición de Unión Canaria de 
Centro (STC 72/1989) y el recurso de inconstitucionalidad interpuesto por el Defensor del Pueblo (STC 225/1998); y Murcia: recurso de amparo interpuesto por D. Luis Ruipérez Sánchez como candidato del Partido Cantonal (STC 193/1989). Todos desestimados por el Tribunal Constitucional como ya se ha quedado explicado en apartados anteriores.

$Y$ es que como advierte MARTínez Sospedra (2001), "a diferencia de lo que ocurre en el caso del Congreso, la norma es que la cláusula sí tenga capacidad de exclusión, en unos casos por ser notablemente más elevada como el caso canario, en otros porque aun no siéndolo el mayor tamaño de la circunscripción sí posibilita que la cláusula excluya (Parlament de Catalunya)".

\section{Elecciones locales}

Como es sabido el Sistema Electoral Local regulado en el Capítulo IV Título III, IV y V de la LOREG, lo conforman las elecciones municipales (arts. 176 a 200), elecciones a los Cabildos canarios (art. 201) y la elección de Diputados Provinciales (arts. 202 a 209). (Vid. PENDÁs, 1997) y (BARAS Y BOTELLA, 1996).

Las elecciones municipales se regulan en el Capítulo IV del Título III de la LOREG remitiéndose a la fórmula electoral empleada para las Elecciones al Congreso (D'Hondt) pero especificando que la circunscripción electoral lo constituirá cada término municipal estableciendo además la relación entre el número de residentes y el número de concejales, y la barrera electoral que según su artículo 180 se fija en el 5 por 100 de los votos válidos emitidos en cada circunscripción. Régimen general que no es aplicable ni a los Municipios de menos de 100 habitantes que funcionan en concejo abierto, $\mathrm{ni}$ a los que tengan una población comprendida entre 100 y 250 habitantes y que serán elegidos de acuerdo con lo establecido en el artículo 148 LOREG.

La misma regulación, también sobre la barrera electoral, se aplica a la elección de los Consejeros Insulares al Cabildo con la excepción de que la circunscripción electoral es la Isla (art. 201.3).

En relación con la afectividad real de la barrera en este tipo de elecciones, MARTínez SOSPEDRA destaca que, salvo en los casos de concejo abierto y en las elecciones de los micromunicipios que se efectúan mediante escrutinio mayoritario, la barrera del $5 \%$ sí es efectiva debido a la elección con método d'Hondt en distrito único. 


\section{Elecciones al Parlamento Europeo}

Las elecciones al Parlamento europeo son las únicas en el ordenamiento electoral español en las que no se aplica ningún tipo de barrera de exclusión. Esta variante unida al método de escrutinio D'Hondt aplicado en circunscripciones donde se reparten más de 60 escaños y a la circunscripción única, hacen del sistema un sistema en el que, como han explicado BARAS y BotelLA, se "potencia al máximo la proporcionalidad de los resultados». Precisamente de ello se han beneficiado sobre todo partidos pequeños como la Agrupación de Electores Ruiz Mateos, que con el 3,84 por 100 de los votos obtiene dos escaños en las elecciones europeas de 1989 (BARAS Y BOTELLA).

\section{REFERENCIAS BIBLIOGRÁFICAS}

Las citas que se han efectuado a lo largo del texto, corresponden a los siguientes autores y obras:

E. Álvarez CONDE, "Los principios del Derecho Electoral», RCEC; núm. 9, Madrid 1991.

BACHOFF, O.: "Derecho electoral y derecho de los partidos en la República Federal Alemana", en Boletín Mexicano de Derecho Comparado, Universidad Nacional Autónoma de México, año XII, núm. 37, enero-abril 1980.

Baras, M. y Botella, J.: El Sistema Electoral, Tecnos, Madrid, 1996.

Bravo de Laguna, J. H.: Comentarios al Estatuto de Autonomía de Canarias, dir. J. Varona Gómez-Acebo, Marcial Pons e Instituto Administración Pública de Canarias, 1996.

De CaRreras, F. Y Vallés, J. M., Las Elecciones, Ed. Blume, Barcelona, 1977.

Fraile Clivillés, M.: "Los Sistemas Electorales", IV Jornadas de Derecho Parlamentario, Congreso de los Diputados, Madrid, 1997.

FERNÁNDEZ SEGADO, F.: Aproximación a la nueva normativa electoral, Dikinson, Madrid, 1986. Estudios de Derecho Electoral, Ediciones Jurídicas, Perú, 1997.

García Roca, J.: Cargos Públicos Representativos. Un estudio del artículo 23.2 de la Constitución, Aranzadi, Pamplona, 1998.

LÁZARO Rı́L, A: «Proporcionalidad e igualdad en los sistemas electorales autonómicos", REDC, núm. 59, Mayo-Agosto 2000.

LÓPEZ AgUILAR, J. F.: «Los problemas de constitucionalidad del sistema electoral canario (La insoportable "permanencia" de una Disposición Transitoria)", REDC, núm. 51, Septiembre-Diciembre 1997.

LOPEZ GARRIDO, D.: «Intervención en el debate sobre "La reforma del Regimen Electoral General" ", Cuadernos y Debates núm. 49, CEC, Madrid 1994.

MASCLET, J. C.: Droit Electoral, Paris, 1989.

MARTÍNEZ CUADRADO, M.: "Bases, principios y criterios para la reforma de la Ley Electoral de 1977", en Revista de Política Comparada, núm. 6, 1981. 
Martínez Sospedra, M.: "Las cláusulas de barrera en la Jurisprudencia del TC", Revista General de Derecho, 2001.

MONTERO, J. R.: «El debate sobre la reforma electoral: rendimientos, criterios y propuestas de reforma", REP, núm. 95, Enero-Marzo, 1997.

Montero, J. R., LLERA, F. J. y TORCAL, M.: “Sistemas electorales en España: una recapitulación", Revista Española de Investigaciones Sociológicas, núm. 58, Madrid, 1992.

Nicolas MUÑIz, J.: "El modelo de un sistema cuasi-mixto: el caso alemán», en El proceso electoral, DE EsTEBAN y otros, Labor, Barcelona, 1977, págs. 247 y ss.

NoHLEN, D.: Leyes Electorales y Sistemas de Partidos, CITEP, Madrid, 1977 y "Sistemas Electorales del Mundo", CEC, Madrid, 1981.

PEndÁs Garcia, B.: "El sistema electoral en las Elecciones Locales", Reflexiones sobre el Régimen Electoral General, IV Jornadas de Derecho Parlamentario, Congreso de los Diputados, Madrid, 1997.

Punset, E. en el Prólogo a D. Rae y V. Ramirez: El sistema electoral español, Macgraw-Hill, Madrid 1993.

RAE, D. W.: Leyes electorales $y$ sistemas de partidos politicos, CITEP, Madrid 1977.

RAE, D. y Ramírez, V.: El sistema electoral español, MacGraw-Hill, Madrid, 1993.

Rius RuLL, F.: «Barreras de exclusión en los sistemas electorales de las Comunidades Autónomas», en Reflexiones sobre Régimen Electoral General, IV Jornadas de Derecho Parlamentario, Congreso de los Diputados, Madrid, 1997.

SÁnChez NavarRo, A. J.: "Constitución, igualdad y proporcionalidad electoral», Cuadernos y Debates, núm. 76, CEPC, Madrid, 1998.

SolozABAL, J. J.: Intervencion en el debate sobre "La Reforma del Regimen Electoral General", Cuadernos y Debates, num. 49, CEC, Madrid 1994.

Torres Del Moral, A.: "Jurisprudencia del Tribunal Constitucional en materia electoral". Acotaciones crítica, RDP, núm. 41, 1996.

VV.AA: "La reforma del Régimen Electoral General", Cuadernos y Debates, núm. 49, CEC, Madrid, 1994.

VALLÉS, J. M.: «Sistema electoral y democracia representativa: Nota sobre la Ley Orgánica del Régimen Electoral General de 1985 y su función polítican, $R E P$, núm. 53, septiembre-Octubre 1986.

VALLÉS, J. M. y BoSCH, A.: Sistemas electorales y gobierno representativo, Ariel, Barcelona, 1997.

VIDAL PRAdo, C.: El sistema Electoral Español, Método Ediciones, Granada, 1995.

YANes HerReros, A.: "Los sistemas electorales para las Asambleas Legislativas de las Comunidades Autónomas", en Reflexiones sobre Régimen Electoral General, IV Jornadas de Derecho Parlamentario, Congreso de los Diputados, Madrid, 1997. 